\title{
Implementation of Constraint Satisfaction Problem Methods on Course Scheduling in High School
}

\author{
Sugiarto $^{1 *}$, Mohammad Idhom ${ }^{2}$, Ronggo Alit $^{3}$, Akhmad Fauzi $^{4}$ \\ ${ }^{1,2,3}$ Department of Informatics \\ ${ }^{4}$ Department of Economic Management \\ Universitas Pembangunan Nasional "Veteran" Jawa Timur \\ Surabaya - Indonesia \\ *sugiarto.if@upnjatim.ac.id
}

\begin{abstract}
Course scheduling in high schools is a complex and complicated activity to solve. Complexity can be seen from the distribution of course, teachers, time, and also classrooms in one teaching and learning activity. In the subject scheduling process there are several things that must be considered. First, there is a schedule where the teacher cannot teach, second, subject scheduling is expected to be evenly distributed on the day for each class, Third, course scheduling will be more complicated, if it involves parallel classes, fourth, there are certain points that use laboratory space must be scheduled in the laboratory room, this requires an optimization method that can be applied to work on this subject scheduling model. Heuristic methods usually produce good solutions or solve complex problems. In solving the problem, Constraint Satisfaction Problem (CSP) is an approach to solving problems with the aim of finding conditions or objects that meet some requirements or criteria in the constraint satisfaction problem (CSP) method. The components contained in the Variable Constraint Satisfaction Problem are that the container can be filled with various values, constraints are rules specified to set values can be loaded into variables, or a combination of variables. Scheduling so that the subject can initially be made easier when all these constraint variables are met.
\end{abstract}

Keywords- Scheduling, High School, Constraint Satisfaction Problem (CSP)

\section{INTRODUCTION}

High School is currently experiencing many developments and additions to several course. The more developed and the many subjects that exist in the school resulted in the difficulty of the academic part to make scheduling of subjects for each teacher in the school. Scheduling is an efficiency effort that is carried out to manage various kinds of resources for multiple activities. In solving scheduling problems, computers are devices that are widely used to provide optimal solutions. One form of activity that requires scheduling is lecture activities. The complexity of applying lecture scheduling algorithms is high.
Planning and scheduling techniques have recently seen essential advances thanks to the application of constraint satisfaction models and tools. Most real-world problems can be cast as highly coupled planning and scheduling problems, where resources must be allocated to optimize overall performance objectives. Therefore, solving these problems requires an adequate mixture of planning, scheduling and resource allocation in competing for goal activities over time in the presence of complex state-dependent constraints. [1]

By going through an effective scheduling process, the use of space, time and human resources can be optimized and can avoid the occurrence of clashes between courses, educators and time caused by constraints. In the obligation of a teacher there is a limit to the minimum number of hours he must teaching, based on Minister of Education and Culture Regulation (Permendikbud) Number 23 of 2017 regarding School Day, in Permendikbud, a teacher is obliged to carry out his teaching workload for 40 hours in 5 (five) days in 1 (one) week.

In the case study that will be applied to high schools there are still many teachers who come from outside the school, which makes scheduling subjects difficult, because they have to adjust an empty schedule based on the subjects they use, so it requires several variables that can accommodate it, by using the Constraint Satisfaction Problem (CSP), there will be several approaches to variable distribution, in the Satisfaction session will collect data for variables that have a narrow limit, while for the Problem can use a variable whose domain is unlimited.

\section{RELATED WORKS}

\section{A. Scheduling}

Scheduling is the time management of an operating activity related to the utilization of resources such as allocating facilities, equipment, and labor by deciding the order of implementation and utilization of these resources for operations. Proper scheduling will maximize the use of every resource in operation, so scheduling is an essential activity in 
planning and controlling the process. The planning stage and implementation phase of scheduling activities include complex time management problems. Stephen R. Covey in his book entitled First thing first: to live, to love, to learn, to leave a legacy, offering a category scheme for various time management approaches, is:

1. The First Generation,

Reminders based on hours and times, but with the possibility of implementation with a computer; can be used to alert someone when there is a task that must be completed.

2. Second Generation,

Planning and preparation based on calendars and agenda books including destination settings.

3. Third Generation,

Planning, prioritizing, controlling (using a personal agenda, paper-based, computer, or PDA-based) activities based on daily schedules. This approach requires the use of a certain amount of time to clarify values and priorities. There are several ways to set priorities, is:

a) ABC Analysis.

b) Pareto Analysis.

c) Eisenhower Method.

d) POSEC Method

4. Four Generation

Become efficient and proactive in using the equipment above; placement of goals and roles as an arrangement of elements of the preferred system and activities above urgent interests. [5]

In line with the development of information technology, the efficiency of the complex scheduling problem resolution approach also continues to increase.

\section{B. Constraint Satisfaction Problem (CSP) Method}

Constraint satisfaction is related to the problem of finite domains and is usually referred to as Constraint Satisfaction Problems (CSP) while constraint solving uses variables that are unlimited domains (infinite domains) so that they are more complex and must be solved by non linear equations, algebra or numerical methods and not just combination and searching. CSP functions to obtain values from variables that meet all constraints. Furthermore, in this paper, the author only discusses the constraint programming and constraint satisfaction in a limited domain [2]

To solve the Scheduling problem using constraint satisfaction, we need to formulate it as a Constraint satisfaction Programming (CSP) problem. A CSP-problem can be defined as:

1. A set of variables $X=\{x 1, \ldots, x n\}$.

2. For each variable $x i$, a finite set $\mathrm{Di}$ of possible values (its domain).

3. A set of constraints restricting the values that the variables can simultaneously take.

The solution of a CSP is obtained through systematic searching of all values of possible variables based on existing constraints. In this case, the searching method to get a solution can be divided into two main groups, namely through partial solution (partial value assignments) and complete value assignments of all variables stochastic. In constraint programming is also known as the term propagation constraint, which is to cut or shorten the search by using existing constraints. Each constraint has a filtering algorithm that can reduce the number of domains of variables in the constraint, by removing values that are not possible to be a solution to the problem. This algorithm is called every time a domain of a constraint variable changes and this change is propagated to the domain of other variables and so on.

To cover the lack of local propagation (incompleteness/lack of shortage), grouping some constraints is done into a special constraint called global constraint. Global constraints are a collection of several small constraints treated as a larger unit (combined). For example are constraints "all different, cumulative, cycle, etc." [3]

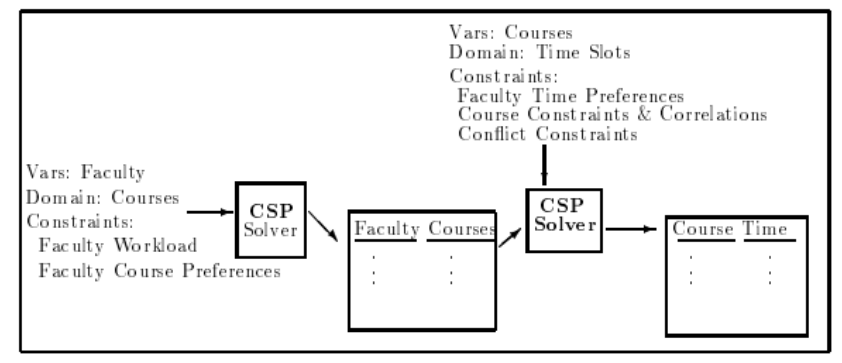

Fig. 1 CSP Method [5]

\section{Constraint Optimization}

The standard of constraint satisfaction technique is to only look for possible solutions, but this can be expanded to get optimal solutions. To get the optimal solution, use the Constraint Satisfaction Optimization Problem (CSOP).

The quality of the solution is usually measured based on a function called the objective function. CSOP consists of a standard CSP and an optimization function that maps each solution to its numerical value. Most industrial applications use this CSOP concept. The most widely used algorithm to get the optimal solution in CSOP is Branch and Bound (B \& B). This B \& B algorithm uses a heuristic function that maps partial labeling to a numerical value with a depth-first search algorithm to get a solution. In the search process, if the domain value passes through the existing constraint, the subtree under partial labeling is trimmed to propagate. The most optimal solution based on constraints is chosen as the solution [3]

The problem is to find any combination of choices that is valid or to prove that no such combination exists, and this problem is NP-complete (i.e., when it is both in NP and NPhard), in general. In a CSP, each decision to be made is called a variable, each possible choice for each decision is called a value, the interactions between decisions are called constraints, and combinations of options are called assignments. These terms are further described in the following definitions.

As a local search algorithm progresses, it is possible that it will consider the same complete assignment more than once, 
which means that local search is not systematic. If a solution exists, but a local search algorithm cannot find it before the limit on time or the number of assignments tested is exceeded, the algorithm will not produce the correct result, which means that (when such limits are imposed) local search is not sound. Alternatively, if no limits are imposed, a local search algorithm could search the same subset of complete assignments repeatedly without ever terminating, so (without any limits imposed) local search is not comprehensive. Because a local search algorithm cannot be both sound and complete, it is impossible for local search to prove that no solution exists for a given instance of CSP. The effectiveness of local search increases with the number of solutions to the given problem. [6]

Using the application, the initial stage of schedule preparation is data input. In this stage, details about the scheduling entities are entered. Most scheduling preferences and constraints depend on these inputted parameters. Accordingly, the application offers a module for easy management of these data. Users use the module to manually check entries before plotting schedules. However, in the implementation of this work, problems occurred because of inconsistent or redundant data inputted by the unit registrars. Physically the same rooms or subjects were given different names in every college. The same problem was encountered in the scheduling implementation at Purdue University [7].

\section{METHODOLOGY}

The scheduling of these subjects makes a major subject of every teaching and learning activity, in high schools on many subjects that are still difficult to choose and put into a schedule, this is because teachers come from outside the school, so there are some conditions where teachers it can be in accordance with the schedule that can be used.

\section{A. Course Scheduling System Design}

ER-Diagrams are used to describe the relationships between entities involved in the system [8], with Entity Relationship Diagrams can make it easier for users in this case the person who designed the database to make changes in the database at any time. The design of ERD in scheduling the subjects of this research is shown in Fig 2.

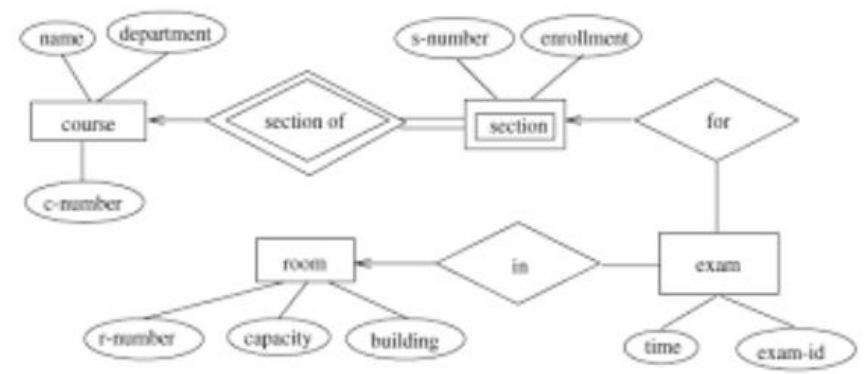

Fig 2 ERD Course Scheduling
Database system design is made using the Logic Data Model. From the design there are eight main tables namely period, class, block, det_block, maple, det_mapel, teacher, det_guru and lab tables. Fig 3.2 describes the eight database tables complete with fields and primary and secondary keys that are used to connect each table.

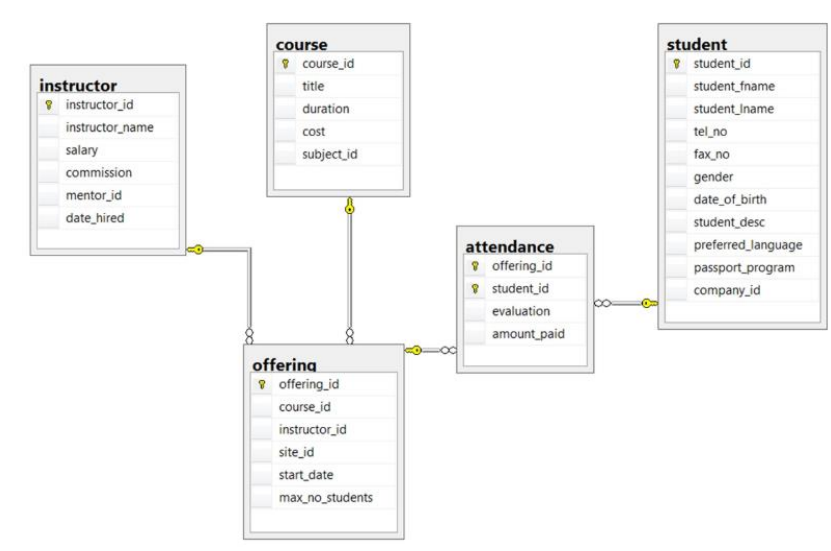

Fig. 3. Database Design and Structure

In Fig 3 above explains that the core process of CSP scheduling lies in the schedule table, wherein the schedule table relates to several tables to be able to create a method that can later run with the appropriate conditions required.

\section{B. Database System}

In Figure 2 ER-Diagram above there are six entities that are interrelated, namely the teacher entity that has four attributes, entity subjects which consist of four attributes, majors entities consisting of two attributes, student entities composed of two attributes, time entities consists of four attributes, and a class entity consisting of three attributes. In addition, on ERDiagrams there are also three relations namely teaching relationship comprising three attributes, relationships take consists of three attributes and relations have consisted of four attributes. After creating ER diagrams, the next step was the realization in the form of the table correspond to the entities and attributes that have been designed in the ER diagram. Relation table comes with a description of the data type and length of data used. In addition, required the determination of the primary key and foreign key attributes defined. Every related entity describes the relationship cardinality. Table relation of scheduling the subjects indicated in Figure 4, with the ER Diagram in Fig 2 facilitates the maintenance of the system on the later to be able to modify and repair the database if there is a mistake in the making of the application later, the database makes a subject that is very important in Constraint Satisfaction Problem (CSP) application, some of the CSP process also becomes a calculation the calculation and stored in the database.

\section{Scheduling Algorithm Flowchart}

Flowchart is a more detailed description of each step of the program. This flowchart shows each step of the program or 
procedure in the right order when occur. The form of a flowchart of these applications can be seen in Fig 5. Fig 5 explained that the input code majors, academic year and semester desperately needed at the time the program is run. As for the initialization time teaching and learning have been set as many as six a day from Monday to Saturday. Because in a day's time is implemented to teach as many as eight-time (at 07:00-07:45, 07:45- 08:30, 08:30-09:15, 09:30-10:15, 10:1511:00, 11:00-11:45, 12:15-13:00 and 13:00-13:45) and Friday five-time (except the clock 11:00-11:45, 12:15-13:00 and 13:00-13:45 because of the implementation of the Friday prayer), then the total time it takes is 45 teaching time.

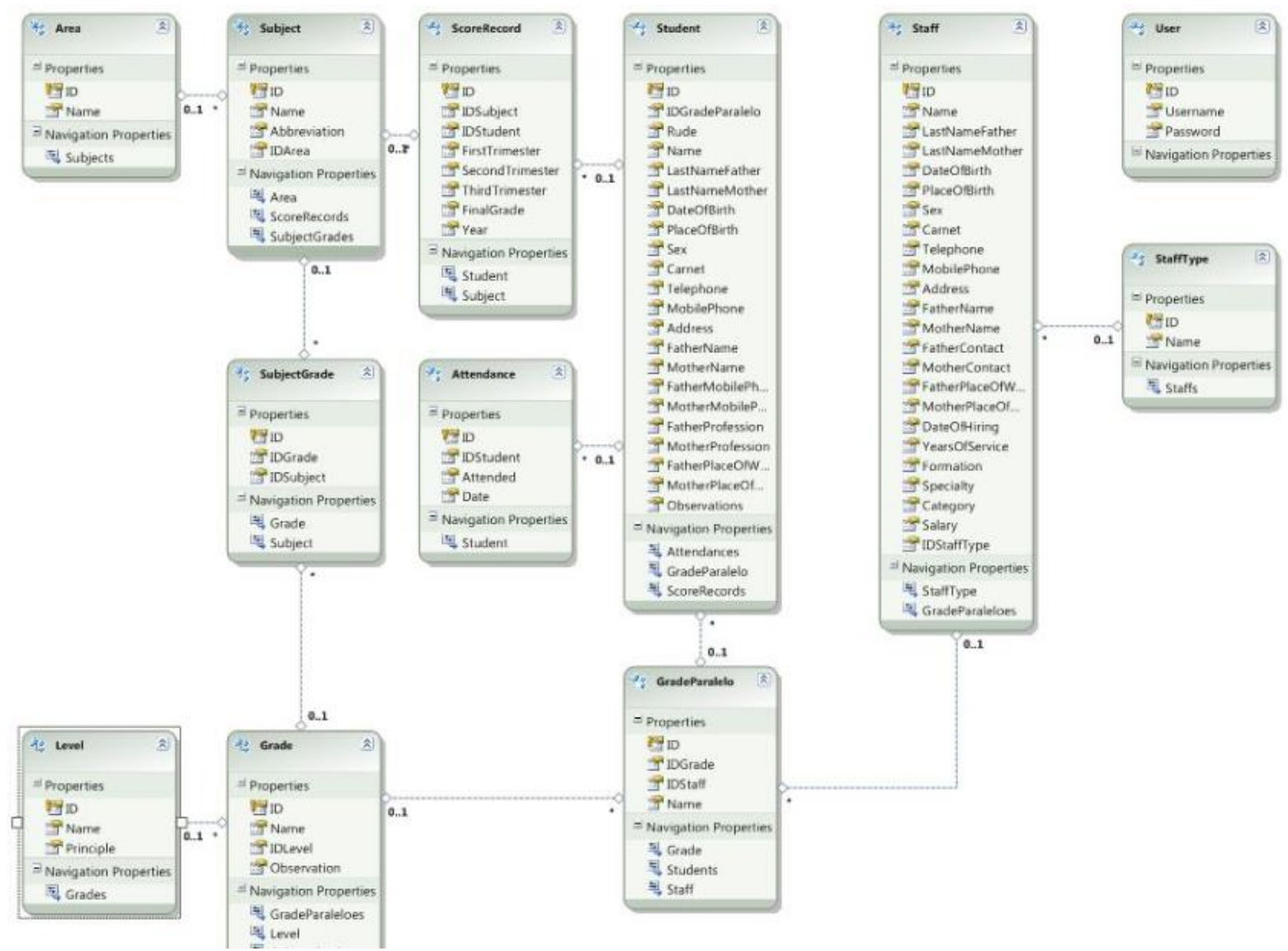

Fig 4. Scheduling Management Course Database

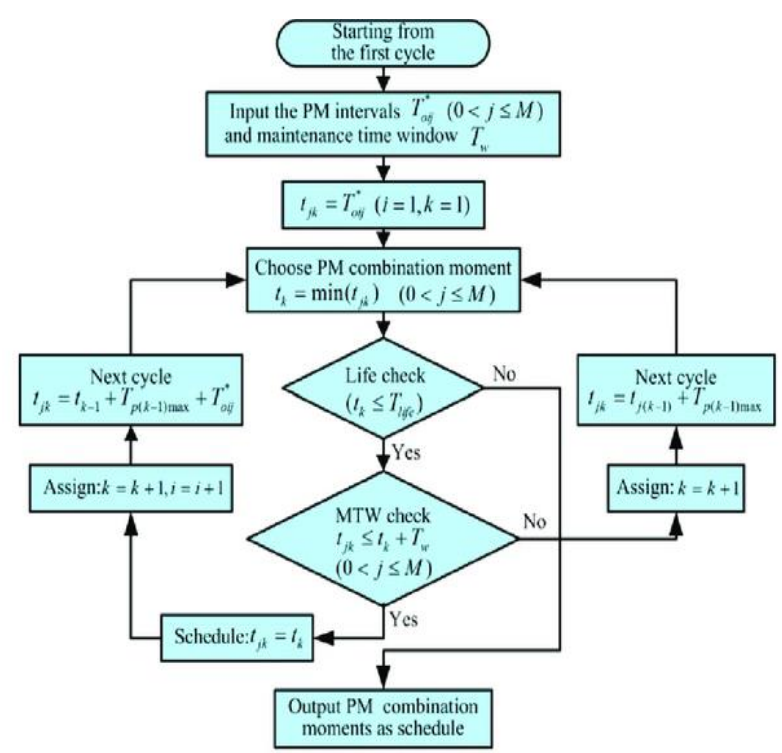

Fig. 5. Scheduling Algorithm Flowchart
As for computer majors here for example (KOM) and the amount of space an eight-room with details of theory and lab capacity load from any room can be seen in table 1 below:

\section{TABLE I. ROOM AND ROOM CAPACITY}

\begin{tabular}{|c|l|l|}
\hline No & \multicolumn{1}{|c|}{ Name of the Room } & Room Capacity \\
\hline 1 & Class Room 1 & 40 \\
\hline 2 & Class Room 2 & 40 \\
\hline 3 & Class Room 3 & 40 \\
\hline 4 & Class Room 4 & 40 \\
\hline 5 & Computer Lab & 36 \\
\hline 6 & Language Lab & 36 \\
\hline 7 & Chemistry Lab & 24 \\
\hline
\end{tabular}

In TABLE I, data for the space allocated for the Computer Department (KOM). For the amount of space theory and lab there are as many as seven rooms while the number of classrooms in the majors KOM 4 classrooms. Therefore, of course, the amount of room is not a sufficient number of existing rombel in KOM, then arranged in such a manner by the system to the existing shortfall allocated to perform the 
working practices of the industry (internship job). In the early stages, all course based on departments, semester and academic year, and the number of students who have the subjects shown separately. The application then displays the number of the room with its capacity. Then, the number of combinations from the room and the amount of time into learning schedule slot for each of the subjects. Furthermore, every subjects taken at random (random) based on Constraint Satisfaction Problems (CSP) Algorithms and distributed into the slots of the earlier study schedule. If the slot is empty, then the schedule of subjects is on that slot. Whereas if the slot is not empty then do reschedule process. This process continues until all the subjects filled slot on the schedule of study and there is no slot that is found with the subjects. The following is an algorithm for the scheduling of process route for each subject by using Constraint Satisfaction Problems Algorithm.

\section{IV.RESULT AND DISCUSSION}

\section{A. Constraint Satisfaction Problems Algorithm}

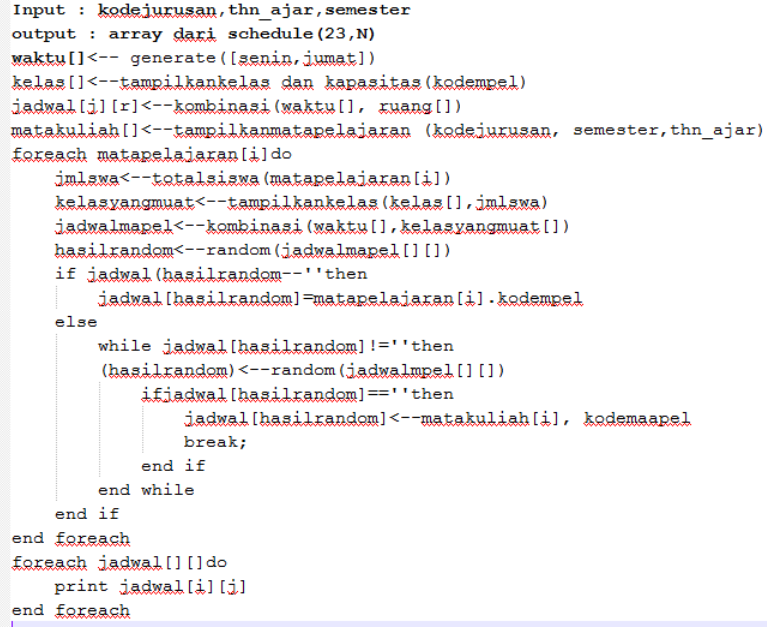

Fig 6. Constraint Satisfaction Problems Algorithm Code

In Figure 6, the initial process undertaken is combining the possibility of time and the available class to the slot assignment schedule for lessons. This process can be shown by using the SQL command that can be seen through the pieces of the program in Fig 6.

\section{Pseudocode 1}

SELECT m.kodemapel, m.mapel, a.jmlswa,

IF (m.bobot>3, 2, 1) AS jmIMasuk

FROM matapelajaran AS $m$

JOIN (

SELECT d.kodemapel, d.jm/swa

FROM mengajar as $d$

WHERE d.kodejurusan='01' AND

d. semester $=$ '3' AND d.jm/swa $>=7$

ORDER BY d.jm/swa DESC

) AS a ON m.kodemapel=a.kodemapel
On Pseudocode 1 above the display algorithm csp all subjects presented by departments, semester, and academic year certain.

Next algorithm scrambles the subjects to be distributed into the slots of the schedule of lessons. If the number of students who follow subjects more than the capacity of the room, then the algorithm to find other suitable room with a capacity of the room. The process of randomization and distribution for each subject can be run through Pseudocode 2 .

\section{Pseudocode 2}

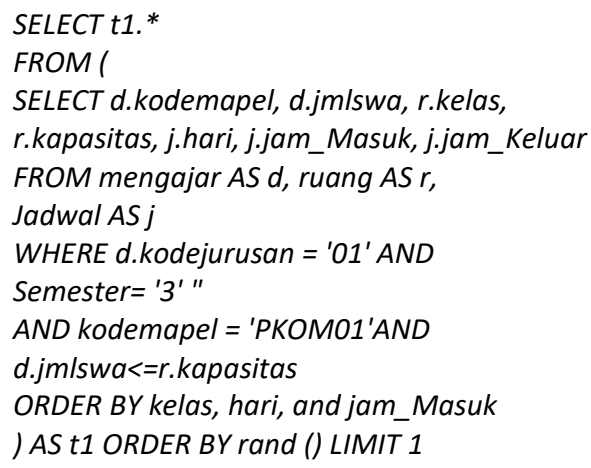

At Peseudocode 2 above shows, one example of allocating the subjects "productive KOM" with code lessons PKOM01 "PKOM01" distributed over the results of the application of randomization. Suppose the number of classrooms of students who participate in this lesson are 36 students and the amount of room that accommodate the number of students is 2 , as well as the number of sessions of study time, is 45 hours of lessons with a total of 6 days KBM, there are 90 the possibility of time that can be allocated to subjects PKOM01. If the assumed time to PKOM01 with the number of subjects 6 hours on these lessons and was carried out on Monday at 07:00-12:00, the space Lab 1. If the results of that combination is not empty or are scheduled for other subjects, then schedule for this course should be random back until it finds a load of time and space. Random processes continue until all subjects already scheduled in all possible time provided.

\section{B. Implementation System}

On the test system Constraint Satisfaction Problem (CSP) problems in the classroom parallel where there is more than 1 class for the same subjects 1 . In the schedule the complete subjects, some data settings must be made in advance, i.e., Period, classes, Subjects, teachers, teachers ' Details and blocks of time. When the regulatory process such data is already done, then the process of scheduling core can be done. Figure 2 display is at the core of the scheduling process, do scheduling, the schedule made first schedule specific subjects complete with schedule and teacher laboratory practice.

Special course of the schedule, then makes a schedule of general subjects (course). The placement of public course schedule tailored to the priorities of the order in any subjects. Fig 7 is the process of creating a schedule of public course, in 
the process first option is placed in the order of most courses the beginning.

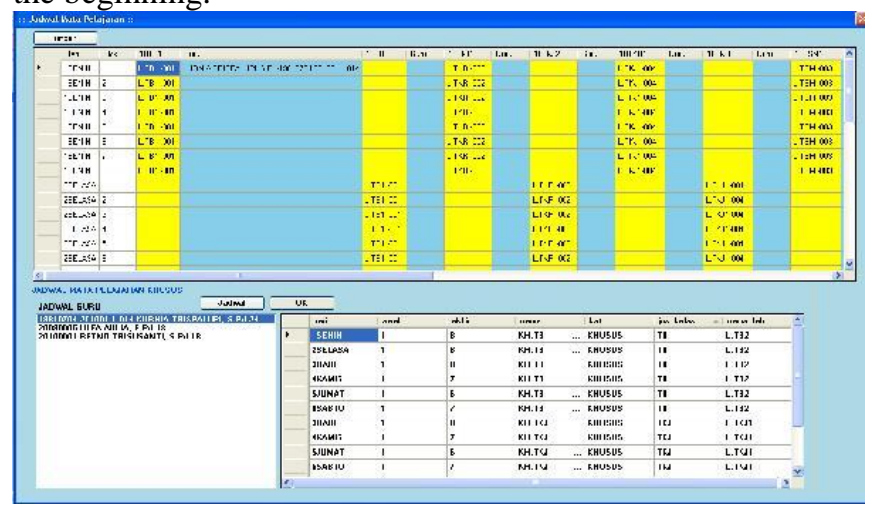

Fig 7. Course Scheduling

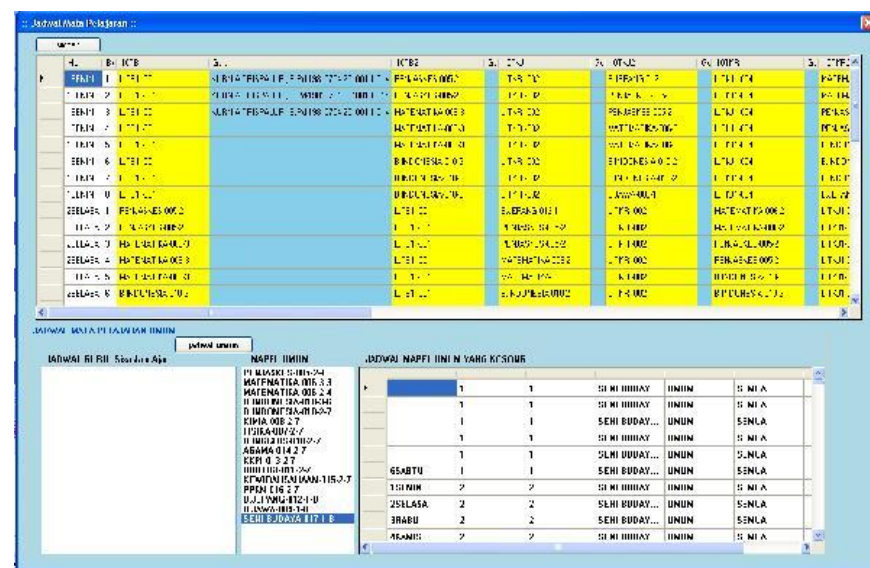

Fig 8. Course Scheduling CSP Algorithm

The result of the schedule of subjects is complete; it can be seen from the report results schedule of subjects for the next process. This application later aka outer tone form of the subjects schedule report users can see every class schedule from Monday-Friday complete with teachers in those subjects. So found on the model set of relationships between teachers, as well as room matapelajaran class can be modeled with a Venn diagram as in Fig 9.

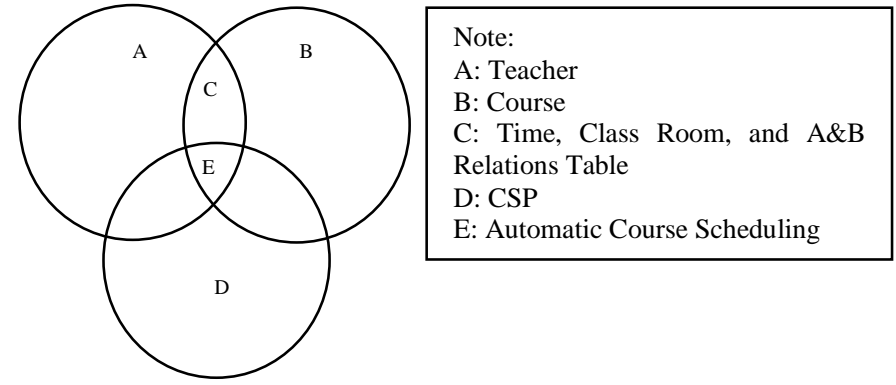

Fig 9. Venn Diagram with CSP Algorithm

\section{CONCLUSION}

Based on the analysis and implementation has been done then it can be summed up as follows:

1. The number of Classrooms that can be maximized based on the amount that can inhabit.

2. Schedule the use of spaces between the competing with one another at the same time can be minimized.

3. A teacher teaching Schedule clashed in the same day can be minimized

4. the Constraint Satisfaction Problem (CSP) can reduce the similarity data from various elements of the main sources of information for making a course scheduling.

\section{REFERENCES}

[1] R. Bartak, M.A. Salido, F. Rossi, "Constraint Satisfaction Techniques in Planning and Scheduling,' Journal of Intelligent Manufacturing, 21(1), pp. 5-15, February 2010.

[2] R. Bartak, Foundations of Constraint Satisfaction, http://kti.mff.cuni.cz/ bartak/, accessed on June 29, 2005

[3] M. Dewi, "Constraint Programming," Faculty of Computer Science, Indonesia University, 2005

[4] R. Bartak, Foundations of Constraint Satisfaction, http://kti.mff.cuni.cz/ bartak/, accessed on June 29, 2005.

[5] S. Covey, A.R. Merrill, R.R. Merrill, First Things First: To Live, to Love, to Learn, to Leave a Legacy, New York: Simon and Schuster, 1994.

[6] P.J. Schwartz, Constraint Optimization Literature Review, Aberdeen MD: ORSA Corporation, November 2015.

[7] K. Murray, T. Muller, H. Rudova, Modeling and Solution of a Complex University Course Timetabling Problem, Retrieved from http://wendang.baidu.com/view/060d78f8770bf78a65295429.html, 2006

[8] Kusrini, Database Design and Management System, Yogyakarta: Andi, 2007 . 